\title{
Tumour detection and outcomes of surveillance screening in SDHB and SDHD pathogenic variant carriers
}

\author{
Gemma White ${ }^{1}$, Anand Velusamy ${ }^{1}$, Samantha Anandappa', Michael Masucci@1, Louise A Breen', Mamta Joshi', \\ Barbara McGowan ${ }^{1,2}$, Johnathan G H Hubbard $\mathbb{1}^{3}$, Rupert Obholzer ${ }^{4}$, Dimitra Christodoulou ${ }^{5}$, Audrey Jacques ${ }^{5}$, \\ Philip Touska ${ }^{5}$, Fahim-UI Hassan ${ }^{6}$, Louise Izatt ${ }^{7}$ and Paul V Carroll ${ }^{1,2}$ \\ 'Department of Diabetes and Endocrinology, Guy's and St Thomas' NHS Foundation Trust, London, UK \\ ${ }^{2}$ Faculty of Life Sciences and Medicine, King's College London, London, UK \\ ${ }^{3}$ Department of Endocrine Surgery, Guy's and St Thomas' NHS Foundation Trust, London, UK \\ ${ }^{4}$ Department of Ear, Nose and Throat Surgery, Guy's and St Thomas' NHS Foundation Trust, London, UK \\ ${ }^{5}$ Department of Radiology, Guy's and St Thomas' NHS Foundation Trust, London, UK \\ ${ }^{6}$ Department of Nuclear Medicine, Guy's and St Thomas' NHS Foundation Trust, London, UK \\ 7Department of Clinical Genetics, Guy's and St Thomas' NHS Foundation Trust, London, UK \\ Correspondence should be addressed to A Velusamy: anand.valusamy@gstt.nhs.uk
}

\begin{abstract}
Objective: Succinate dehydrogenase subunit (SDHx) pathogenic variants predispose to phaeochromocytoma and paraganglioma (PPGL). Lifelong surveillance is recommended for all patients to enable prompt detection and treatment. There is currently limited evidence for optimal surveillance strategies in hereditary PPGL. We aim to detail the clinical presentation of PPGL in our cohort of non-index SDHB and SDHD pathogenic variant carriers.

Methods: Retrospective analysis of medical and genetic records from a single tertiary referral centre identified $S D H B$ or $S D H D$ pathogenic variants in 74 non-index cases (56 $S D H B$ and $18 S D H D$ ). Surveillance screening for asymptomatic relatives consisted of annual plasma metanephrine measurement and whole-body MRI with contrast at 3-5 yearly intervals.

Results: Twenty-three out of 74 non-index patients (10 SDHB and 13 SDHD) were diagnosed with PPGL, 17 patients through surveillance screening (24 tumours in total) and 6 diagnosed prior to commencement of cascade screening with symptomatic presentation. MRI with contrast identified PPGL in 22/24 screen-detected tumours and $5 / 24$ tumours had elevated plasma metanephrine levels. Penetrance in non-index family members was 15.2 and $47.2 \%$ for SDHB carriers and 71.6 and $78.7 \%$ for SDHD carriers at age of 50 and 70 years, respectively.

Conclusion: Surveillance screening with combined biochemical testing and imaging enables early detection of PPGL in asymptomatic relatives with SDHx pathogenic variants. The presence of disease at first screen was significant in our cohort and hence further multicentre long-term data are needed to inform counselling of family members undergoing lifelong surveillance.
\end{abstract}

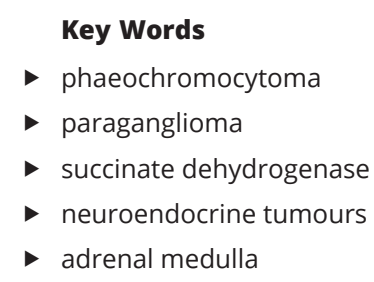

Endocrine Connections (2022) 11, e210602 https://ec.bioscientifica.com https://doi.org/10.1530/EC-21-0602

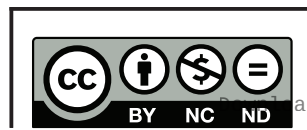

This work is licensed under a Creative Commons Attribution-NonCommercial-NoDerivatives 4.0 Internationab ticense.ifica.com at 04/25/2023 11:13:36PM 


\section{Introduction}

Phaeochromocytomas and paragangliomas (PPGLs) are neuroendocrine tumours arising from neural crest tissue. Phaeochromocytoma (PCC) arises from the adrenal medulla and paragangliomas (PGLs) from autonomic ganglia, usually arising from the sympathetic chain within the thorax, abdomen and pelvis or the parasympathetic ganglia located within the head, neck and upper thorax (1). Adrenal and sympathetic tumours are often catecholamine-releasing in nature, whereas parasympathetic tumours predominantly tend to be biochemically inert or occasionally noradrenaline or dopamine-dominant releasing (2).

PPGLs are now recognised as the most heritable tumours, with current literature demonstrating up to $40 \%$ of cases are attributable to a genetic cause (3). More than 19 genes have to date been identified to predispose susceptibility to both syndromic and isolated forms (4). Amongst the identified genes, pathogenic variants in the succinate dehydrogenase ( $\mathrm{SDH})$ subunits genes $(\mathrm{SDHx})$ account for almost half of these hereditary cases (2). The penetrance and phenotype have been shown to vary greatly between different $S D H$ subunit pathogenic variants. A recent large study cited penetrance at $22.5 \%$ for $S D H B$ and $50.0 \%$ for $S D H D$ carriers, respectively, at age of 60 years (5). In terms of location, $S D H B$-related tumours occur most commonly along the sympathetic chain, whereas $S D H D$ related disease predominantly affects the parasympathetic ganglia of the head and neck (6). Risk of malignancy has been shown to be highest in $S D H B$-related disease, at a rate of $\sim 30 \%$ (7), compared to about $8 \%$ in SDHD carriers (8). Multifocal disease, however, is more common in $S D H D$ patients, occurring in $55-60 \%$ of cases, compared to $20-25 \%$ of $S D H B$ patients (9).

Current guidelines recommend genetic testing where a heritable cause is suspected, such as in bilateral adrenal disease, extra-adrenal PGLs, metastatic disease or positive family history; however, there is a growing evidence base to advocate genetic screening in all PPGL cases (10). In solitary low-risk PCC in the absence of family history, genetic testing is currently funded by NHS England when diagnosed in patients under 60 years of age or if immunohistochemical testing demonstrates loss of SDH staining (11). If a germline pathogenic variant in a PPGLsusceptibility gene is identified, cascade genetic testing of relatives is recommended to identify family members at risk of tumour development and thus allowing enrolment into surveillance screening programmes (12).

Since the discovery of $S D H x$-related PPGL, screening recommendations for asymptomatic pathogenic variant carriers have been debated. Consensus of the literature recommends annual clinical review with blood pressure measurement and metanephrine level assessment (13). In terms of imaging, early studies advocated the use of CT, ${ }^{123}$ I-MIBG or PET-CT due to enhanced specificity for PPGL $(14,15)$. However, more recent suggestions from The Endocrine Society (13) and Tufton et al. (6) advocate minimising radiation by using MRI as the initial screening tool. The recommended frequency of imaging also varies, ranging from every 1 to 5 years $(6,14,16,17)$. An international consensus statement published in 2021 aimed to better standardise the current screening protocols (18). For the initial screen, they recommended clinical review, plasma metanephrine measurement, whole-body MRI consisting of MRI from the skull base to pelvis and PET-CT to detect undiagnosed disease in the mediastinum, where functional imaging has been demonstrated to perform better than anatomical imaging - sensitivity of somatostatin receptor scintigraphy is $61.5 \%$ vs $46.2 \%$ for anatomical imaging (15). Following an initial negative screen, annual clinical review with metanephrine level assessment and whole-body MRI every 2-3 years has been recommended for all $S D H x$ variants. For carriers who do not develop tumours during the course of follow-up, the consensus was to reduce the screening frequency to every 5 years after 70 years of age and cessation of screening at 80 years of age.

Given the limited evidence base underlying screening recommendations, further penetrance and longitudinal analyses of tumour development are needed to ensure adequate tumour detection in asymptomatic $S D H x$ pathogenic variant carriers, whilst minimising radiation exposure and patient burden. Risk stratification using several components, including pathogenic variant (both germline and somatic), histology, biochemistry, imaging and various other metabolomics should be considered for a precise, cost-effective long-term follow-up and management. We, therefore, present our surveillance screening data from a single tertiary centre cohort of patients found to carry pathogenic variants in $S D H B$ and $S D H D$ genes. The main objective of this study is to inform the relatives regarding the likelihood of disease at cascade screening and also during subsequent follow-up.

\section{Methods}

Patients presenting with PPGL to a single tertiary centre from January 2000 to December 2020 reported to carry an $S D H x$ pathogenic variant were identified retrospectively 
from genetic databases, medical records and multidisciplinary meeting (MDM) records. Cascade screening in the form of genetic testing followed by whole-body MRI with contrast and plasma metanephrines in mutationpositive cases was offered to all first-degree relatives (youngest being 8 years in $S D H B$ and 13 years in $S D H D$ ). Demographic information, tumour characteristics, screening history and results were extracted from medical records.

\section{Genetic testing}

Genetic testing was performed by our regional genetics services. Index cases underwent testing for 10 PPGLsusceptibility genes (RET, VHL, SDHA, SDHB, SDHC, SDHD, SDHAF2, TMEM127, MAX and $F H$ ) with sequence analysis of coding regions performed, plus Sanger sequencing to confirm any identified variants. Multiplex ligation-dependent probe amplification analysis was also performed for all exons of $V H L$ and $S D H B / C / D$ to identify large deletions or duplications. For relatives, targeted genetic testing for the variant identified in the proband was performed. Variants were reported as pathogenic, benign or of uncertain significance (VUS). Variants were cross-referenced with the ClinVar database (19) to confirm pathogenicity according to American College of Medical Genetics and Genomics guidelines (20). Patients with VUS were excluded from the analysis to prevent underestimation of penetrance.

PPGL diagnosis was made from histological analysis of excised tumour specimen or from a combination of biochemistry and anatomical/functional imaging when the tumour remained in situ. Metastatic disease was defined as the presence of PPGL tissue in non-chromaffin organs. Multifocal disease was classified as more than one tumour affecting any chromaffin organ. Metastatic/multifocal disease was classified as synchronous (detected 0-6 months after primary tumour diagnosis) or metachronous (detected more than 6 months after primary tumour).

\section{Surveillance screening protocol}

Baseline surveillance screening for patients with no history of PPGL consisted of a full clinical review with blood pressure measurement, measurement of plasma metadrenaline (MA), normetadrenaline (NMA), and 3-methoxytyramine (3-MT) and cross-sectional imaging using whole-body MRI with contrast and diffusion-weighted imaging sequences (more recently included) to increase sensitivity for the detection of SDH-related PPGLs (21).
Plasma metanephrines were measured by liquid chromatography-tandem mass spectrometry based on a published method adapted to incorporate measurement of 3-MT (13). Biochemical testing of metanephrines was considered positive if any one or more of MA, NMA or 3-MT levels were elevated above the upper reference limit. MRI was considered positive if the radiologist identified a lesion anatomically suspicious of PPGL requiring investigation. Additional investigations to confirm diagnosis, such as functional imaging, were decided upon at the MDM, as were treatment strategies once the diagnosis was established. If initial screening was negative, follow-up surveillance for relatives consisted of clinical review with measurement of plasma metanephrines on an annual basis, with contrastenhanced MRI of the neck, thorax, abdomen and pelvis every 3-5 years, based on MDM outcome and patient preference. Discussion at MDM involved assessment of disease presentation in the index case and other affected family members, considering high-risk features for aggressive disease outlined in the literature including metastatic disease, multifocality, primary tumour size ( $>5 \mathrm{~cm}$ for PCC and $>3 \mathrm{~cm}$ for PGL), pathogenic variant, extra-adrenal location and younger age at diagnosis $(<40$ years) $(22)$.

\section{Statistical analysis}

Statistical analysis was performed using GraphPad Prison 8.0 (GraphPad Software, Inc.). Kaplan-Meier curves were used to calculate penetrance. To avoid ascertainment bias, index cases were excluded from penetrance calculations, as is recommended by recent literature (5).

\section{Ethical approval}

Ethical approval was sought from the NHS Health Research Authority in April 2018 (IRAS Reference Number: 205985). This study was carried out in accordance with the Declaration of Helsinki.

\section{Results}

Sixty-one index patients with a diagnosis of phaeochromocytoma or paraganglioma were identified as carriers of a germline pathogenic $S D H B$ or $S D H D$ variant after undergoing genetic testing between January 2000 and December 2020. From these index cases, 141 relatives were reported to carry an $S D H B$ or $S D H D$ pathogenic variant. Data were available for 74 relatives (56 SDHB and $18 S D H D$ ) 
who were followed up in our centre. The remaining 67 relatives were not followed up at our centre or declined follow-up.

\section{Outcomes of surveillance screening}

From 74 relatives identified through genetic records, 6 patients had prior diagnosis of PPGL when examining medical records ( $1 S D H B$ and $5 S D H D)$. The remaining 68 relatives had no prior history of PPGL and were entered into the surveillance screening programme, with patient characteristics shown in Table 1 . Median age at start of screening in our cohort was higher for SDHB patients at 40 years compared to 25 years for SDHD patients. Median screening duration was 3 years for both groups. The cumulative total of screening data available for this cohort was 272 patient-years.

PPGL was diagnosed in 17 of the 68 (25\%) screened relatives. The median age at diagnosis was 43 years ( 49 years for $S D H B, 26.5$ years for $S D H D)$, with the youngest patient found to have a tumour aged 15 years (SDHD) and the oldest aged 74 years (SDHD). Detailed clinical information for these patients is available in Table 2 .

For patients carrying $S D H B$ pathogenic variants, screening detected tumours in $9 / 55$ patients (16.4\%), all of which were diagnosed during first screen. MRI detected all SDHB-related tumours except for two mediastinal PGLs. One patient (patient 3) was found to have a PCC on MRI, with a synchronous mediastinal PGL which was later detected upon further imaging with ${ }^{123}$ I-MIBG. Another patient (patient 4) was found to have elevated NMA levels and subsequently underwent ${ }^{18}$ F-FDG-PET imaging which identified the mediastinal PGL. Two other tumours visible on MRI, one abdominal PGL (patient 2) and one HNPGL (patient 9) demonstrated NMA excess.
For patients carrying SDHD pathogenic variants, tumours were detected in 8/13 patients (61.5\%) during screening. All SDHD-related lesions were visible on surveillance MRI and metanephrines (NMA and 3-MT) were elevated in only one patient with 2 synchronous abdominal PGLs (patient 13). Disease in seven relatives was picked up on the first screen, with one further patient diagnosed with bilateral SDHD-related HNPGL (patient 11) 1 year after an initial negative MRI and non-elevated metanephrines. Four patients (patients 11, 13, 16 and 17) were found to have synchronous tumours at first screen. Patient 11 was also found to have two further biochemically inert vagal PGLs detected on follow-up surveillance MRI 9 years after the initial screen-detected carotid body PGLs. Review of previous imaging did not reveal evidence of these vagal PGLs, meaning they represent true late metachronous lesions.

Tumour size detected on MRI ranged from 7 to $40 \mathrm{~mm}$ in maximal diameter. Five tumours remain in situ (patients 6, 7, 9, 14 and 16) and are under active surveillance with a current duration of follow-up over 2-9 years. All in situ tumours remain biochemically inert and have shown absent or minimal $(<2 \mathrm{~mm})$ growth on MRI. Metastatic disease, either at the time of diagnosis or during follow-up was not detected in any relatives during the course of the study. No other SDHx-related tumours (RCC, GIST and pituitary adenoma) were detected during surveillance screening. Two patients were found to have small $(<2 \mathrm{~cm})$ incidental adrenal adenomas on cross-sectional imaging and functionality were excluded. No other incidental findings were reported.

\section{Penetrance}

Penetrance calculations for 56 non-index patients with $S D H B$ pathogenic variants and 18 relatives with SDHD

Table 1 Characteristics of 68 non-index cases with no prior history of PPGL undergoing surveillance screening.

Male $(n, \%)$
Age at initial screening
Duration of screening
Number of screens
Patients diagnosed with PPGL $(n, \%)$
Number of tumours
Phaeochromocytoma
Mediastinal PGL
Abdominal PGL
Head and neck PGL
Screening modality detected by $(n, \%)$
Cross-sectional imaging
Metanephrine testing

\begin{tabular}{c} 
SDHB $(n=55)$ \\
\hline $28(50.9 \%)$ \\
$40(8-71)$ \\
$3(1-15)$ \\
$2.5(1-7)$ \\
$9(16.4 \%)$ \\
10 \\
$1(10 \%)$ \\
$3(30 \%)$ \\
$2(20 \%)$ \\
$4(40 \%)$ \\
$8(80 \%)$ \\
$3(30 \%)$
\end{tabular}

\begin{tabular}{c} 
SDHD $(n=13)$ \\
\hline $8(61.5 \%)$ \\
$25(13-74)$ \\
$3(1-14)$ \\
$2(1-12)$ \\
$8(61.5 \%)$ \\
14 \\
0 \\
0 \\
$4(28.6 \%)$ \\
$10(71.4 \%)$ \\
$14(100 \%)$ \\
$1(7.1 \%)$
\end{tabular}



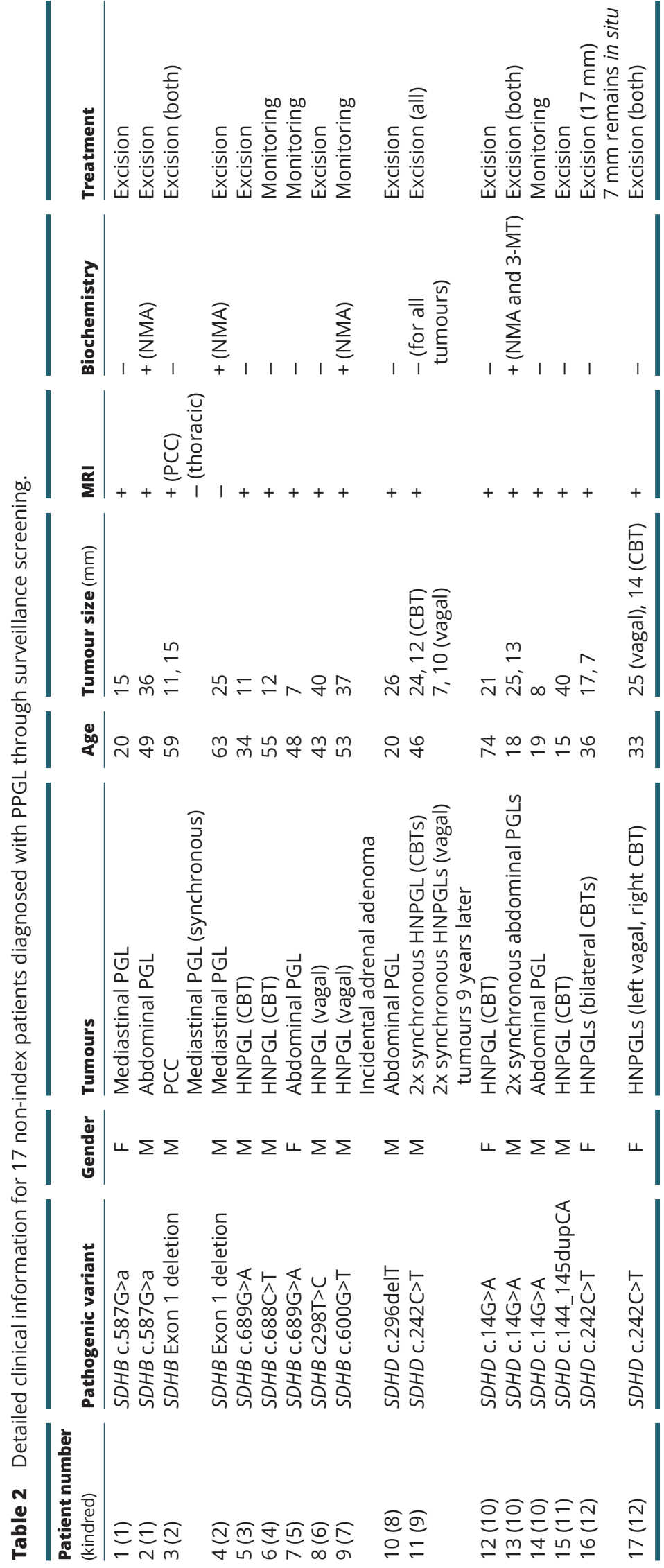
pathogenic variants can be seen in Fig. 1. Penetrance at age 50 was $15.2 \%$ in $S D H B$ and $71.6 \%$ in $S D H D$, respectively. Penetrance at age 70 was $47.2 \%$ in $S D H B$ and $78.7 \%$ in $S D H D$, respectively.

\section{Discussion}

We analysed the outcomes of our SDHx screening programme in a single tertiary referral centre and calculated the penetrance of $S D H B$ and $S D H D$ pathogenic variants in our cohort, aiming to improve understanding of disease development and progression. Through this, we aim to strengthen the current evidence-base advocating for standardisation of lifelong surveillance screening protocols for carriers of $S D H x$ pathogenic variants.

For relatives enrolled in our screening programme, PPGLs were detected in carriers of both $S D H B$ and $S D H D$ pathogenic variants. For SDHB, 16.4\% (9/55) of relatives were diagnosed with PPGL at first screen. These findings are comparable with previously reported screening programmes. In a study by Greenberg et al. (23), tumours were detected in 15\% (29/188) of their cohort of asymptomatic relatives with $S D H B$ pathogenic variants. Jochmanova et al. (24) cited a rather lower detection rate of $9.5 \%(23 / 241)$ in family members during the initial screen. Tufton et al. found a slightly higher detection rate, with $25.0 \%$ (15/60) of their screened cohort developing tumours, with 10/15 identified on first screen and 5 further tumours picked up 2-6 years following the initial screen (25).

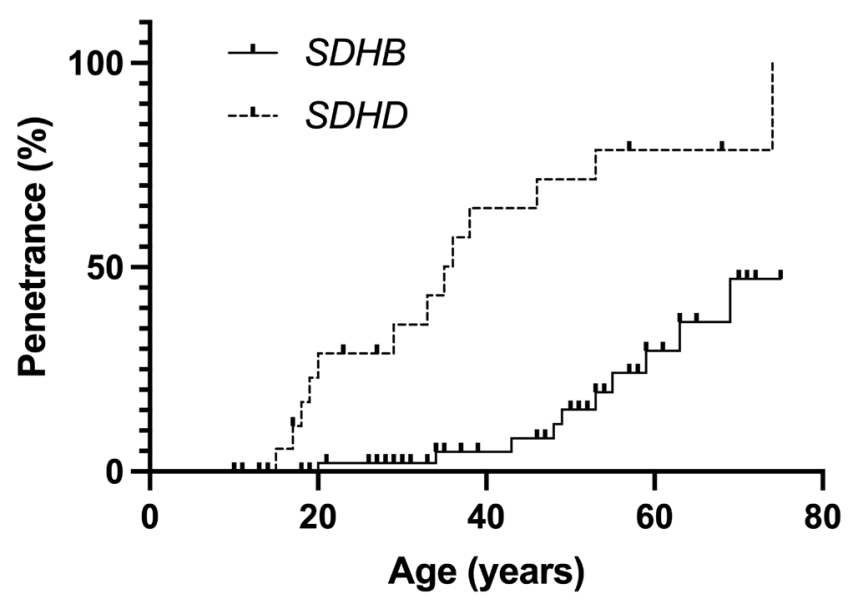

\section{Figure 1}

Penetrance of PPGL in non-index cases. Penetrance in 56 patients harbouring SDHB pathogenic variants was 15.2 and $47.2 \%$ at age of 50 and 70 years, respectively. Penetrance in 18 patients harbouring SDHD pathogenic variants was 71.6 .1 and $78.7 \%$ at age of 50 and 70 years, respectively.
The median follow-up period was 5.7 years, compared to 3 years in our study. The reduced screening duration in our study highlights the need for longer screening duration to fully evaluate the incidence of PPGLs following an initial negative screen.

For SDHD, surveillance screening detected more tumours than $S D H B$, with $61.5 \%(8 / 13)$ of screened relatives developing tumours, 7/8 of which were detected on first screen. Two further HNPGLs were also identified in one patient 9 years after the initial tumour detection. The detection rate in our cohort is similar to Heestermann and colleagues, who found tumours in 59.5\% (28/47) of asymptomatic SDHD carriers at first screening (26), but is higher than findings from Greenberg et al. where $40 \%(14 / 35)$ of relatives with SDHD pathogenic variants developed tumours over a mean screening duration of 2 years (24).

Our surveillance screening protocol included both cross-sectional imaging, using MRI with contrast, and biochemical testing of plasma metanephrines, as suggested by current guidelines $(13,18)$. International consensus from Amar et al. (18) recommends additional use of PET-CT at initial screening to increase sensitivity of detecting mediastinal PGLs, although this is not standard practice at our centre unless in the context of aggressive disease in other family members or elevated metanephrines with no PPGL identifiable on MRI. MRI with contrast and diffusion-weighted imaging has proven to be an effective screening tool in our patient cohort and avoids the use of ionising radiation. However, there were two small mediastinal SDHB-related PGL which were not visualised on initial MRI, but were detected upon follow-up functional imaging. Identification of small $(<1 \mathrm{~cm})$ mediastinal PGLs on MRI can be challenging due to motion artefact and spatial resolution, emphasising the importance of biochemical assessment and consideration of alternative imaging modalities. The inclusion of functional imaging at baseline screening, as recently recommended, may have aided detection in these cases and should be explored in future analyses of screening protocols. The clinical significance of identifying a sub-centimetre non-secretory mediastinal PGL should be carefully weighed against radiation exposure and cost-effectiveness in these patients needing lifelong surveillance. HNPGLs were predominantly biochemically inert, consistent with previous data that suggests $S D H$ related HNPGL are often better detected by imaging, especially in early stages $(25,27,28)$. We observed a low proportion of catecholamine-releasing tumours in our cohort compared to data reported in the literature for

This work is licensed under a Creative Commons Attribution-NonCommercial-NoDerivatives 4.0 Internationad ticense.ifica.com at 04/25/2023 11:13:36PM 
hereditary disease (29). This suggests that surveillance imaging is effective in detecting tumours before they become biochemically active, allowing intervention before symptoms and clinical sequelae of catecholamine excess occurs. Despite this, regular measurement of plasma metanephrines remains a valuable tool in assessing risk of metastatic disease, especially in sympathetic PGLs (30).

We acknowledge few limitations in our study including the sample size of 56 SDHB and 18 SDHD nonindex carriers, which is relatively small for penetrance calculations to be performed when compared to recent larger studies, such as Andrews et al. with 371 and 67 SDHB and $S D H D$ pathogenic variant carriers, respectively (5). Our cohort may also not be representative due to the singlecentre study design and status as a regional referral centre for PPGL. Additionally, as a specialist centre for ENT, there is also a referral bias, where the presentation of multifocal PPGL with predominant head and neck location and families with high penetrance may be overrepresented in our cohort. Therefore, larger multi-centre studies that include a collation of other specialist units throughout the UK are needed to ensure appropriate representation of the study cohort.

We conclude that an appropriate surveillance strategy is imperative following the diagnosis of germline $S D H x$ pathogenic variant with or without PPGL. The burden of disease is significant at first screen $(16.4 \%$ in $S D H B$ and $54 \%$ in $S D H D$ ) in our cohort. Appropriate imaging helps in early detection of the disease before plasma metanephrines become measurable in the circulation. Hence we also advocate plasma metanephrines over urine measurement in $S D H x$ disease. We also recommend whole-body MRI for anatomical imaging as it avoids the use of ionising radiation in a cohort already requiring lifelong surveillance. Such imaging should typically be contrast-enhanced, with the benefits of improved PGL identification (particularly at the skull base) outweighing the risks of gadolinium retention within the CNS (31). Whole-body MRI may also be supplemented by functional imaging modalities as required during the course of management. We believe that the metastatic potential, particularly in $S D H B$ patients, and potential for multifocality in both $S D H B$ and $S D H D$ patients warrants lifelong surveillance. Additionally, we emphasise periodic whole-body surveillance as opposed to selective imaging in these groups and advocate for management and follow-up in tertiary units under MDM with expertise in PPGLs. It is hoped that through continuing research and larger longitudinal studies, optimal cost-effective tailored surveillance and treatment strategies can be devised in order to improve patient outcomes.

\section{Declaration of interest}

The authors declare that there is no conflict of interest that could be perceived as prejudicing the impartiality of the research reported.

\section{Funding}

This work did not receive any specific grant from any funding agency in the public, commercial or not-for-profit sector.

\section{References}

1 Tischler A, Gill A, Kawashima A, Kimura N, Komminoth P \& Papathomas T. Tumours of the adrenal medulla and extra-adrenal paraganglia. In WHO Classification of Tumours of Endocrine Organs, pp. 179-207. Eds R Lloyd, R Osamura, G Klöppel \& J Rosai. Lyon: International Agency for Research on Cancer, 2017.

2 Turchini J, Cheung VKY, Tischler AS, De Krijger RR \& Gill AJ. Pathology and genetics of phaeochromocytoma and paraganglioma. Histopathology 201872 97-105. (https://doi.org/10.1111/his.13402)

3 Castro-Vega LJ, Lepoutre-Lussey C, Gimenez-Roqueplo AP \& Favier J. Rethinking pheochromocytomas and paragangliomas from a genomic perspective. Oncogene 201635 1080-1089. (https://doi.org/10.1038/ onc.2015.172)

4 Crona J, Taïeb D \& Pacak K. New perspectives on pheochromocytoma and paraganglioma: Toward a molecular classification. Endocrine Reviews 201738 489-515. (https://doi.org/10.1210/er.2017-00062)

5 Andrews KA, Ascher DB, Pires DEV, Barnes DR, Vialard L, Casey RT, Bradshaw N, Adlard J, Aylwin S, Brennan P, et al. Tumour risks and genotype-phenotype correlations associated with germline variants in succinate dehydrogenase subunit genes SDHB, SDHC and SDHD. Journal of Medical Genetics 201855 384-394. (https://doi.org/10.1136/ jmedgenet-2017-105127)

6 Tufton N, Sahdev A, Drake WM \& Akker SA. Can subunit-specific phenotypes guide surveillance imaging decisions in asymptomatic SDH mutation carriers? Clinical Endocrinology 201990 31-46. (https:// doi.org/10.1111/cen.13877)

7 Timmers HJLM, Kozupa A, Eisenhofer G, Raygada M, Adams KT, Solis D, Lenders JWM \& Pacak K. Clinical presentations, biochemical phenotypes, and genotype-phenotype correlations in patients with succinate dehydrogenase subunit B-associated pheochromocytomas and paragangliomas. Journal of Clinical Endocrinology and Metabolism 200792 779-786. (https://doi.org/10.1210/jc.2006-2315)

8 van Hulsteijn LT, Dekkers OM, Hes FJ, Smit JWA \& Corssmit EPM. Risk of malignant paraganglioma in SDHB-mutation and SDHDmutation carriers: a systematic review and meta-analysis. Journal of Medical Genetics 201249 768-776. (https://doi.org/10.1136/ jmedgenet-2012-101192)

9 Benn DE, Robinson BG \& Clifton-Bligh RJ. 15 YEARS OF PARAGANGLIOMA: Clinical manifestations of paraganglioma syndromes types 1-5. Endocrine-Related Cancer 201522 T91-T103. (https://doi.org/10.1530/ERC-15-0268)

10 Casey R, Garrahy A, Tuthill A, O'Halloran D, Joyce C, Casey MB, O'Shea P \& Bell M. Universal genetic screening uncovers a novel presentation of an SDHAF2 mutation. Journal of Clinical Endocrinology and Metabolism 201499 E1392-E1396. (https://doi.org/10.1210/ jc. 2013-4536)

11 UK Genetic Testing Network. National genomic test directory: testing criteria for rare and inherited disease, 2018. (available at: https://www. england.nhs.uk/wp-content/uploads/2018/08/rare-and-inheriteddisease-eligibility-criteria-v2.pdf)

12 Martins R \& Bugalho MJ. Paragangliomas/pheochromocytomas: clinically oriented genetic testing. International Journal of Endocrinology 20142014 794187. (https://doi. org/10.1155/2014/794187) 
13 Lenders JWM, Duh QY, Eisenhofer G, Gimenez-Roqueplo AP, Grebe SKG, Murad MH, Naruse M, Pacak K, Young WF \& Endocrine Society. Pheochromocytoma and paraganglioma: an Endocrine Society clinical practice guideline. Journal of Clinical Endocrinology and Metabolism 201499 1915-1942. (https://doi.org/10.1210/jc.2014-1498)

14 Favier J, Amar L \& Gimenez-Roqueplo AP. Paraganglioma and phaeochromocytoma: from genetics to personalized medicine. Nature Reviews. Endocrinology 201511 101-111. (https://doi.org/10.1038/ nrendo.2014.188)

15 Gimenez-Roqueplo AP, Caumont-Prim A, Houzard C, Hignette C, Hernigou A, Halimi P, Niccoli P, Leboulleux S, Amar L, BorsonChazot F, et al. Imaging work-up for screening of paraganglioma and pheochromocytoma in SDHx mutation carriers: a multicenter prospective study from the PGL.EVA investigators. Journal of Clinical Endocrinology and Metabolism 201398 E162-E173. (https://doi. org/10.1210/jc.2012-2975)

16 Daniel E, Jones R, Bull M \& Newell-Price J. Rapid-sequence MRI for long-term surveillance for paraganglioma and phaeochromocytoma in patients with succinate dehydrogenase mutations. European Journal of Endocrinology 2016175 561-570. (https://doi.org/10.1530/EJE-160595)

17 Eijkelenkamp K, Osinga TE, de Jong MM, Sluiter WJ, Dullaart RPF, Links TP, Kerstens MN \& van der Horst-Schrivers ANA. Calculating the optimal surveillance for head and neck paraganglioma in SDHBmutation carriers. Familial Cancer 201716 123-130. (https://doi org/10.1007/s10689-016-9923-3)

18 Amar L, Pacak K, Steichen O, Akker SA, Aylwin SJB, Baudin E, Buffet A, Burnichon N, Clifton-Bligh RJ, Dahia PLM, et al. International consensus on initial screening and follow-up of asymptomatic SDHx mutation carriers. Nature Reviews: Endocrinology 202117 435-444. (https://doi.org/10.1038/s41574-021-00492-3)

19 Landrum MJ, Lee JM, Benson M, Brown GR, Chao C, Chitipiralla S, Gu B, Hart J, Hoffman D, Jang W, et al. ClinVar: improving access to variant interpretations and supporting evidence. Nucleic Acids Research 201846 D1062-D1067. (https://doi.org/10.1093/nar/gkx1153)

20 Richards S, Aziz N, Bale S, Bick D, Das S, Gastier-Foster J, Grody WW, Hegde M, Lyon E, Spector E, et al. Standards and guidelines for the interpretation of sequence variants: a joint consensus recommendation of the American College of Medical Genetics and Genomics and the Association for Molecular Pathology. Genetics in Medicine 201517 405-424. (https://doi.org/10.1038/gim.2015.30)

21 Tufton N, White G, Drake WM, Sahdev A \& Akker SA. Diffusion-weighted imaging (DWI) highlights SDHB-related tumours: a pilot study. Clinical Endocrinology 201991 104-109. (https://doi.org/10.1111/cen.13980)

22 Nölting S, Ullrich M, Pietzsch J, Ziegler CG, Eisenhofer G, Grossman A $\&$ Pacak K. Current management of pheochromocytoma/ paraganglioma: a guide for the practicing clinician in the era of precision medicine. Cancers 201911 1505. (https://doi.org/10.3390/ cancers11101505)

23 Greenberg SE, Jacobs MF, Wachtel H, Anson A, Buchmann L, Cohen DL, Bonanni M, Bennett B, Naumer A, Schaefer AM, et al. Tumor detection rates in screening of individuals with SDHx-related hereditary paraganglioma-pheochromocytoma syndrome. Genetics in Medicine 202022 2101-2107. (https://doi.org/10.1038/s41436-0200921-3)

24 Jochmanova I, Wolf KI, King KS, Nambuba J, Wesley R, Martucci V, Raygada M, Adams KT, Prodanov T, Fojo AT, et al. SDHB-related pheochromocytoma and paraganglioma penetrance and genotypephenotype correlations. Journal of Cancer Research and Clinical Oncology 2017143 1421-1435. (https://doi.org/10.1007/s00432-017-2397-3)

25 Tufton N, Shapiro L, Srirangalingam U, Richards P, Sahdev A, Kumar AV, McAndrew L, Martin L, Berney D, Monson J, et al. Outcomes of annual surveillance imaging in an adult and paediatric cohort of succinate dehydrogenase B mutation carriers. Clinical Endocrinology 201786 286-296. (https://doi.org/10.1111/cen.13246)

26 Heesterman BL, Bayley JP, Tops CM, Hes FJ, van Brussel BTJ, Corssmit EPM, Hamming JF, van der Mey AGL \& Jansen JC. High prevalence of occult paragangliomas in asymptomatic carriers of SDHD and SDHB gene mutations. European Journal of Human Genetics 201321 469-470. (https://doi.org/10.1038/ejhg.2012.203)

27 Jasperson KW, Kohlmann W, Gammon A, Slack H, Buchmann L, Hunt J, Kirchhoff AC, Baskin H, Shaaban A \& Schiffman JD. Role of rapid sequence whole-body MRI screening in SDH-associated hereditary paraganglioma families. Familial Cancer 201413 257-265. (https://doi.org/10.1007/s10689-013-9639-6)

28 Alrezk R, Suarez A, Tena I \& Pacak K. Update of pheochromocytoma syndromes: genetics, biochemical evaluation, and imaging. Frontiers in Endocrinology 20189 515. (https://doi.org/10.3389/ fendo.2018.00515)

29 Lenders JWM, Pacak K, Walther MM, Linehan WM, Mannelli M, Friberg P, Keiser HR, Goldstein DS \& Eisenhofer G. Biochemical diagnosis of pheochromocytoma: which test is best? JAMA $2002 \mathbf{2 8 7}$ 1427-1434. (https://doi.org/10.1001/jama.287.11.1427)

30 Van Berkel A, Lenders JWM \& Timmers HJLM. Diagnosis of endocrine disease: biochemical diagnosis of phaeochromocytoma and paraganglioma. European Journal of Endocrinology 2014170 R109-R119. (https://doi.org/10.1530/EJE-13-0882)

31 McDonald JS \& McDonald RJ. MR imaging safety considerations of gadolinium-based contrast agents: gadolinium retention and nephrogenic systemic fibrosis. Magnetic Resonance Imaging Clinics of North America 202028 497-507. (https://doi.org/10.1016/j. mric.2020.06.001)

Received in final form 14 January 2022

Accepted 21 January 2022

Accepted Manuscript published online 21 January 2022 https://ec.bioscientifica.com https://doi.org/10.1530/EC-21-0602
(C) 2022 The authors Published by Bioscientifica Ltd
This work is licensed under a Creative Commons Attribution-NonCommercial-NoDerivatives 4.0 elnternationad dicense.ifica . com at 04/25/2023 11:13:36PM 